\title{
Contralateral Prophylactic Mastectomy
}

National Cancer Institute

\section{Source}

National Cancer Institute. Contralateral Prophylactic Mastectomy. NCI Thesaurus. Code C46070.

Surgical removal of a non-diseased breast along with a cancerous one to reduce the risk of future breast carcinoma. 